\title{
Racismo institucional e a saúde da mulher negra: uma análise da produção científica brasileira
}

\author{
Institutional racism and black woman health: an analysis of Brazilian \\ scientific production
}

Beatriz Muccini Costa Oliveira', Fabiana Kubiak'

DOI: $10.1590 / 0103-1104201912222$

RESUMO O Brasil carrega em sua história séculos de escravidão e ideologias racistas que se refletem no quadro de desigualdades sociais na atualidade. Pesquisas revelam que mulheres negras possuem o pior acesso e qualidade de atendimento em saúde, o que seria consequência do racismo institucional. Com base nesses dados, realizou-se revisão de literatura utilizando a metodologia de revisão sistemática com objetivo de levantar a produção científica brasileira referente ao racismo institucional e à saúde da mulher negra, além de analisar como o tema tem sido tratado pelos pesquisadores. Ficou evidenciado que a literatura a respeito permanece escassa, reforçando a necessidade de se abordar o tema racismo nas pesquisas. Apesar de a desigualdade racial se confirmar em todos os artigos analisados, suas conclusões foram diversas e alguns autores interpretaram os dados como consequência apenas da desigualdade econômica. Conclui-se que o debate a respeito do racismo é de fundamental importância no seu combate e que a associação da desigualdade racial à condição econômica é um reflexo do mito da democracia racial que contribui para a manutenção do racismo institucional. É necessária maior sensibilização dos profissionais a respeito do racismo, de forma a que se torne indispensável pensar na categoria racial para obtenção de uma saúde igualitária.

PALAVRAS-CHAVE Racismo. Saúde da mulher. Parto. Violência.

ABSTRACT Brazil carries in its history centuries of slavery and racist ideologies that are reflected in its current social inequalities. Research shows that black women experience the worst access and quality of health care, which would be a consequence of institutional racism. Based on those data, a literature review was applied using the systematic review methodology with the aim to survey the Brazilian scientific production regarding institutional racism and the health of black women, as well as to analyze how the theme has been treated by researchers. It became clear that the literature on the subject remains scarce, reinforcing the need to address the theme racism in further research. Although racial inequality is confirmed in all articles analyzed, their conclusions vary among them, and some authors interpreted data solely as a consequence of economic inequality. We concluded that the debate about racism is of pivotal importance in the fight against it and that the identification of racial inequality with economic condition is a consequence of the racial democracy myth that contributes to the institutional racism

${ }^{1}$ Centro Universitário UniRuy (UniRuy/Wyden) Salvador (BA), Brasil.

biamuccini.c@gmail.com perpetuation. Raising awareness about racism is needed among professionals so that it becomes essential to consider the category 'race' for equal health.

KEYWORDS Racism. Women's health. Parturition. Violence. 


\section{Introdução}

O Brasil vivenciou a escravidão por mais de três séculos. Durante esse período, a desvalorização do negro a partir de suas características físicas e de sua cultura era utilizada como justificativa da dominação branca. Nesse momento, a inferioridade do negro era algo incontestável e o racismo era produzido de forma explícita em razão das normas sociais da época ${ }^{\mathbf{1}, \mathbf{2}}$.

Compreende-se o racismo como um conjunto de ideologias, doutrinas e ideias presentes na sociedade que atribuem inferioridade natural a determinados grupos étnicos e atua como motor de desigualdades que produzem condições precárias de existência da população vitimada ${ }^{3}$. Nos últimos anos, diversas pesquisas têm evidenciado a construção racista na qual se fundamenta o Brasil4-6.

Após a abolição da escravatura, a proclamação da República no Brasil, em 1889, trouxe junto uma nova constituição, que, apesar de garantir a liberdade da população negra, não assegurou seus direitos, de forma a não oferecer reais ganhos materiais ou simbólicos a essa parcela da população, mantendo, assim, 0 status quo da branquitude e a marginalização do negro 4 . Entretanto, por não ter tido em suas constituições direitos civis diferenciados a partir da cor da pele - diferentemente do ocorrido nos Estados Unidos e África do Sul, com os princípios legais separatistas chamados, respectivamente, Jiw Crow e Apartheid -, criou-se a ideia que os conflitos raciais brasileiros foram resolvidos de forma pacífica e, portanto, que o problema do racismo do Brasil teria se solucionado $4,6,7$.

A ideia que os conflitos raciais do país foram resolvidos com o fim da escravidão e com o processo de mestiçagem ocorrido na história brasileira gera o mito da democracia racial, que é a crença popular de que não existem raças no Brasil, portanto, que o racismo não existe ${ }^{4,6,7}$. Como consequência do mito da democracia racial há a recusa no brasileiro em reconhecer sua própria raça e, junto a isso, a ideia de que falar sobre racismo não cabe no contexto nacional $\mathbf{4}^{\mathbf{6}, \mathbf{6}}$. Assim, qualquer tentativa de se falar em negritude ou políticas sociais para negros é vista como uma maneira de segregar a identidade racial do Brasil, o que dificulta ainda mais o combate ao racismo.

Hoje, a sociedade brasileira possui um quadro de desigualdades em que brancos e negros ocupam diferentes espaços sociais. A população negra apresenta piores indicadores sociais, possui menor nível de escolaridade e renda, menor acesso à saúde e serviços sociais, condições mais precárias de moradia e está inserida em piores posições de trabalho ${ }^{8}$. Porém, apesar do visível contraste, o racismo ainda é invisível para os olhos da sociedade, e dados como esses, muitas vezes, são interpretados como fruto da desigualdade econômica e não racial.

Pode-se dizer, então, que o racismo se manifesta de diversas formas na sociedade, e, apesar de suas formas mais explícitas serem proibidas por lei, permanece na estrutura social moldando o comportamento dos indivíduos, como, por exemplo, a forma diferenciada pela qual as instituições tratam os sujeitos. O racismo institucional, definido como "o fracasso coletivo de uma organização para prover um serviço apropriado e profissional para as pessoas por causa de sua cor, cultura ou origem étnica"9(146), possui uma forma sutil de manifestação e se encontra implícito no funcionamento das instituições.

No âmbito da saúde, o racismo institucional acarreta diferenças significativas na pesquisa e no cuidado com doenças predominantemente negras, como, por exemplo, a anemia falciforme. A falta de conhecimento dos profissionais a respeito da doença é marcante e tem como consequência seu diagnóstico tardio, apesar de a descoberta precoce da doença ser um dos principais fatores para um bom prognóstico ${ }^{6,9}$. Além disso, muitas vezes, os sintomas são tratados como dependência química, outro exemplo da estigmatização da população negra. 
O racismo institucional está presente também na prestação de serviços: sua influência vai desde a restrição ao acesso à saúde pela população negra até a relação entre os profissionais de saúde ${ }^{10}$. Identifica-se uma ideologia presente nas redes de assistência à saúde ligada a pensamentos como 'pessoas negras são mais resistentes à dor' ou 'negro não adoece' que tendem a minimizar queixas advindas de pessoas negras e minorar o uso de medicamentos e anestesias, principalmente tratando-se de mulheres nos procedimentos de pré-natal e parto 9,11 .

$\mathrm{O}$ racismo e o sexismo repercutem de diferentes formas na sociedade, implicando restrições específicas dos direitos das populações vitimadas, e, ao se tratar de mulheres negras, considerando, ainda, a ação de um duplo preconceito. A exemplo disso, dados revelam que as mulheres negras possuem menor expectativa de vida e maior índice de morte por transtornos mentais do que as mulheres brancas. Há também a prevalência e reincidência de miomas uterinos e a realização de histerectomia em mulheres negras, além de taxa elevada de mortalidade materna, o que, segundo Domingues et al. ${ }^{12}$, seria um reflexo dos problemas de acesso e pior qualidade de atendimento.

Tratando-se de violência obstétrica, mulheres negras constituem a maioria das mulheres que relatam ter sofrido algum tipo de violência durante a gestação ${ }^{13}$. A violência obstétrica é definida pela apropriação dos processos reprodutivos das mulheres por meio de um tratamento desumanizado que inclui o abuso da medicalização e patologização de processos naturais. A violência obstétrica praticada pelos profissionais de saúde retira das mulheres a autonomia sobre seus próprios corpos e sua capacidade de decidir livremente a respeito de seus processos reprodutivos ${ }^{\mathbf{1 4}}$. Enquanto a violência obstétrica tornou-se um tema de estudo recorrente nos últimos anos, pouco se fala sobre a interseccionalidade das opressões e seus reflexos na saúde da mulher negra. $\mathrm{O}$ desconhecimento das demandas da mulher negra a respeito de sua saúde configura mais uma forma de discriminação e reforça as estruturas racistas das instituições.

Diante dessas discussões, delineou-se este estudo, que consiste em revisão bibliográfica a respeito do tema 'saúde da mulher negra e racismo institucional', cujo objetivo foi levantar a produção científica brasileira referente ao racismo institucional e à saúde da mulher negra e analisar como o tema tem sido tratado pelos pesquisadores.

\section{Metodologia}

A revisão de literatura se utilizou da metodologia de revisão sistemática proposta por Gil15 e foi realizada a partir das plataformas Capes (Coordenação de Aperfeiçoamento de Pessoal de Nível Superior), SciELO (Scientific Electronic Library Online) e Google Acadêmico, utilizando como palavras-chaves: racismo, racismo institucional, mulheres negras, violência obstétrica e parto. Durante o processo de obtenção de dados, os descritores foram modificados, assim como o limite temporal desta pesquisa, que passou de cinco para dez anos, sendo posteriormente retirada pela escassez de resultados encontrados. Excluíram-se da revisão artigos que abordavam apenas a violência obstétrica sem interseccionalidade com a raça e os que traziam a questão racial sem abordar gênero e saúde.

A leitura do material consistiu de quatro etapas. A primeira, chamada por Gil ${ }^{15}$ de leitura exploratória, teve como objetivo a verificação da obra consultada em relação ao interesse de pesquisa. Aqui foram selecionados os artigos de acordo com os descritores e critérios de exclusão. A leitura seletiva, segunda etapa da leitura, se caracterizou pela maior profundidade de seleção do material em relação ao tema de pesquisa.

Após a seleção dos textos, foi feita a leitura analítica, que teve como finalidade ordenar e classificar as informações para que fosse possível a obtenção de respostas aos problemas da pesquisa. Os artigos selecionados foram 
categorizados a partir do ano de publicação, autor, base de dados em que foi encontrado, local da pesquisa, fonte de publicação, abordagem e delineamento. Também foram registradas as variáveis das pesquisas, seus objetivos e conclusões. Na última etapa do processo de revisão, na leitura interpretativa, foram conferidos os resultados da etapa anterior e relacionados com o objetivo da pesquisa.

\section{Resultado e discussões}

Os resultados da pesquisa bibliográfica mostraram a escassez de textos que versam sobre a saúde da mulher negra. Dos 19 artigos referentes ao tema, o primeiro foi publicado em 2000; sete, no ano de 2016; dois cada, nos anos de 2004, 2012 e 2013; e um artigo por ano em 2000, 2003, 2006, 2014 e 2015.

Gráfico 1: Produção por ano

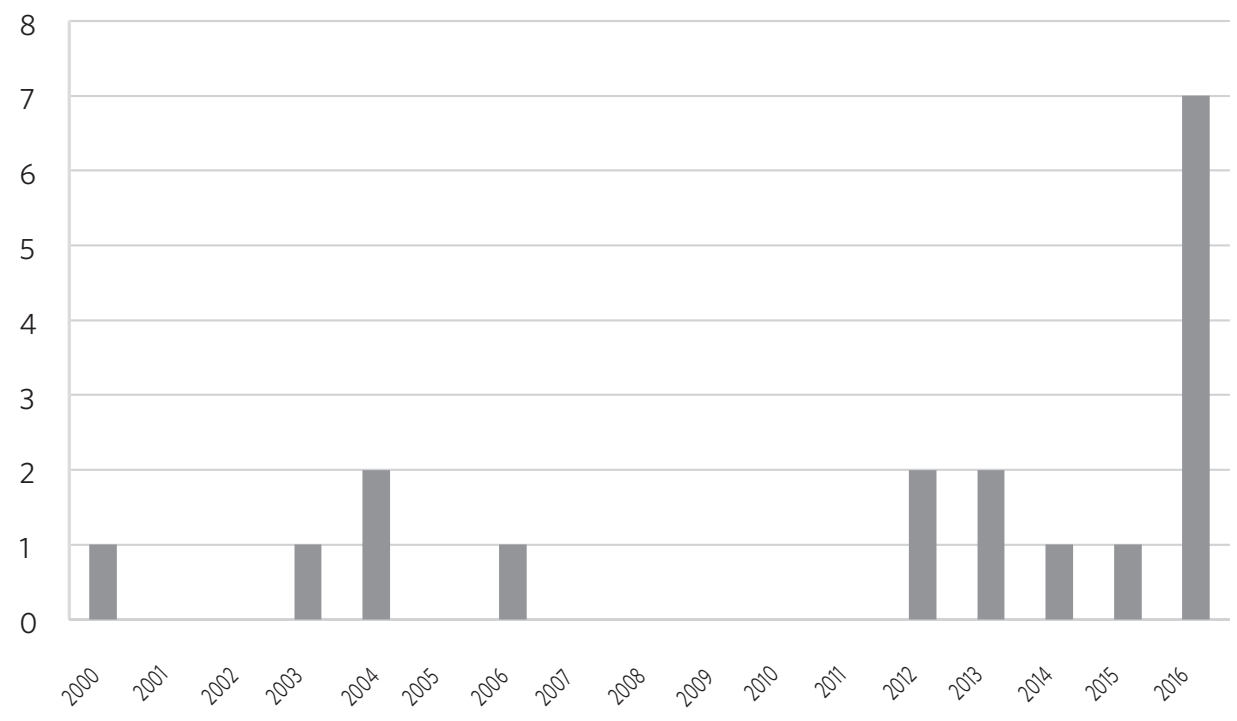

A partir desses dados, pode-se dizer que não só a relação entre racismo e saúde da mulher negra é pouco estudada como também a entrada desse assunto na literatura brasileira é relativamente recente. Embora não seja possível afirmar o motivo da baixa produção científica, esta já era esperada, e número escasso de artigos confirmou o chamado racismo epistêmico evidenciado por $\mathrm{Cruz}^{16}$ e por Werneck $\mathbf{1 0}^{\mathbf{0}}$.

O estudo realizado por $\mathrm{Cruz}^{\mathbf{1 6}}$, em que também foi encontrada escassez de publicações no mesmo tema, interpreta este dado como uma contradição entre o que a academia considera como interessante e digno de pesquisas e os reais problemas vivenciados pela população. A autora salienta, também, que o não reconhecimento das diferenças configura padrões de violência e discriminação e que:

Os resultados deste continuado desconhecimento, isto é, deste racismo epistemológico, sobre aspectos relativos às mulheres negras, em particular, podem ser notados pelos baixos indicadores sociais e de desenvolvimento, assim como pela demora em se estabelecer 
políticas públicas afirmativas na área da saúde e da educação que promovam o bem estar social e a qualidade de vida deste segmento

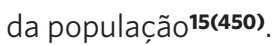

É visível o aumento do número de artigos publicados no ano de 2016, embora não seja possível aferir com certeza o porquê. A primeira explicação possível seria o aumento de pessoas negras na universidade devido a um maior investimento nos programas do governo, como Programa Universidade para todos (Prouni) e Fundo de Financiamento ao Estudante do Ensino Superior (Fies) nos anos de 2013 e 2014, assim como a implantação da lei de cotas no ano de 2012. Entretanto, os artigos foram, em sua maioria, construídos a partir de programas de pós-graduação. Seria possível relacionar com os programas de bolsa para mestrado e doutorado oferecidos pelo governo, porém não foi possível encontrar os dados referentes ao ano de 2016 nas plataformas do Conselho Nacional de Desenvolvimento Científico e Tecnológico (CNPq) e da Capes. Permanece, então, o questionamento sobre o que poderia ter impulsionado a publicação de artigos sobre racismo em 2016.

Das 19 pesquisas realizadas, dez partiram de abordagens quantitativas e, em sua maioria, possuem abrangência nacional. Em 26,3\% dos artigos estudados, os dados utilizados para análise foram retirados de pesquisas pré-existentes, como o Inquérito Nascer do Brasil, Pesquisa Rede Cegonha, Pesquisa Nacional de Amostra por Domicílios (PNAD), dentre outros. Essa informação levou a questionar os motivos pelos quais os pesquisadores preferiram utilizar tais dados em detrimento de trabalhos em campo, e se essa decisão denotaria um distanciamento entre pesquisadores e seu tema de pesquisa.

Uma hipótese para tal distanciamento seria a escolha da metodologia utilizada como reflexo das barreiras encontradas para se abordar o tema racismo. Em outras palavras, as dificuldades para entrar nos serviços poderiam favorecer o direcionamento de pesquisas com a utilização de dados pré-existentes.

Para confirmar essa hipótese, foram analisados os objetivos desses artigos. Observouse que grande parte deles utilizou partes de pesquisas realizadas, de forma que não se pode afirmar que se tratava de dados pré-existentes. Ainda assim, considera-se que tal informação não invalida a possível resistência dos serviços em conduzir pesquisas sobre racismo em suas instalações.

Todos os textos utilizados nesta revisão traziam como problemas centrais questões de saúde da mulher negra, embora apenas dois artigos tivessem como foco a violência obstétrica, reafirmando, mais uma vez, a necessidade de se falar sobre o assunto. $\mathrm{O}$ estudo feito por Lima $^{13}$ revela que apenas $12 \%$ das mulheres negras, de um total de 2.719 mulheres, e $11 \%$ das pardas, de um total de 1.1765 mulheres, presentes na amostra de 23.095 mulheres não sofreram nenhum tipo de violência no atendimento ao parto. Dentre as mulheres que sofreram violência no parto, a proibição do acompanhante no procedimento foi a violência mais praticada.

A pesquisa de d'Orsi et al. ${ }^{17}$, de abrangência nacional, mostra que os percentuais de mulheres que relatam algum tipo de violência, seja ela física, verbal ou psicológica, são maiores entre pretas ou pardas, de menor escolaridade e da região do Nordeste. Em contrapartida, as mulheres brancas de classe A e B possuem melhor índice de satisfação no atendimento à saúde.

Apesar de poucos artigos trazerem a violência obstétrica como tema central do estudo, dados referentes ao tema estão contidos na grande maioria das pesquisas, onde ficou evidenciado que a violência obstétrica está presente desde o pré-natal, dado que já era esperado. O estudo de Belfort, Kalckmann e Batista ${ }^{\mathbf{1 8}}$, realizado no estado do Maranhão, revela que, apesar de ter havido uma melhoria inegável no atendimento ao pré-natal, com fornecimento da quantidade mínima de consultas, não seria garantia de melhoria da assistência, 
uma vez que, a despeito disso, mulheres pretas ainda apresentam altos índices de anemia ferropriva e hipertensão arterial durante o período gestacional. Esses dados evidenciam que o atendimento não está sendo eficaz, que a comunicação necessária para a gestante precisaria ser adequada às suas necessidades, de forma a garantir sua compreensão e maior participação no acompanhamento gestacional.
No que diz respeito à procedência das pesquisas, observamos que a região Sudeste apresentou o maior número de publicações, sete artigos, seguido pelo Nordeste, com cinco publicações. As regiões Sul e Centro-Oeste publicaram, respectivamente, três e dois artigos. Não foram encontrados artigos oriundos da região Norte, e identificamos uma publicação multicêntrica.

Gráfico 2. Produção por região

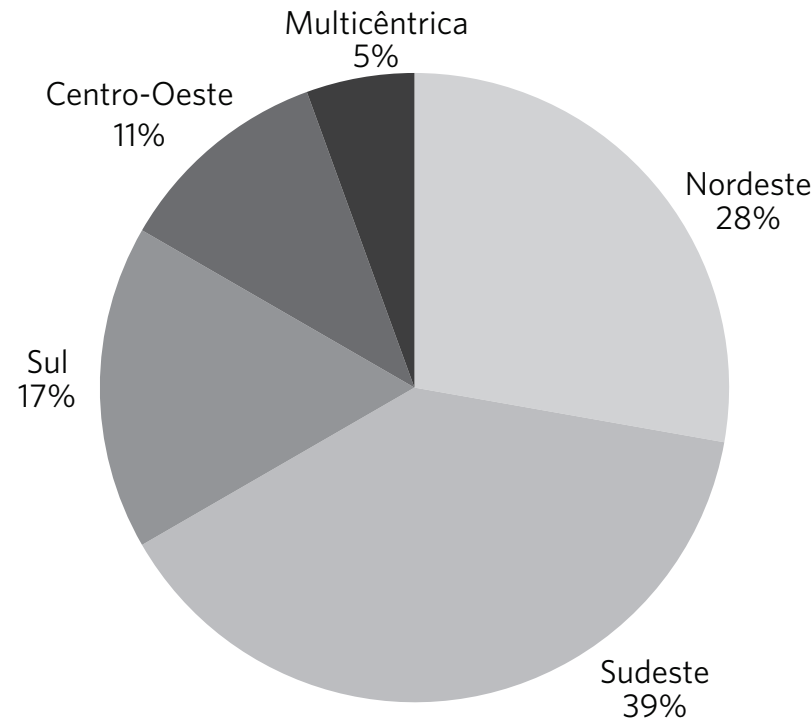

Surpreende a falta de publicações na região Norte e que o Nordeste não se destaque como o maior produtor de pesquisas sobre o tema, visto que a maior parte da população negra brasileira reside no Nordeste. No entanto, é compreensível que o Sudeste se mantenha como polo de produções acadêmicas, uma vez que concentra a maior parte de universidades e instituições de fomento à pesquisa.

Dentre as variáveis utilizadas, todas as pesquisas têm em comum a raça, idade e escolaridade. A situação conjugal aparece como variável em oito pesquisas, a religião, em duas, e a variável renda, em onze artigos. Importa ressaltar que a variável renda exerce impacto sobre o resultado das pesquisas, visto que os autores tendem a levar em consideração que a condição econômica está relacionada à questão da raça. Além disso, entende-se que mulheres negras e de baixa renda estariam mais expostas ao racismo institucional.

Chama à atenção que a religião, apesar de aparecer em duas pesquisas, não foi utilizada na análise desses artigos. Cabe a interrogação sobre a importância ou o impacto da religião na investigação sobre 
o racismo. O baixo número de pesquisas e descarte dessa variável nas discussões leva à conclusão que, de fato, a religião não tem impacto sobre o tema.

Em relação à situação conjugal, não foram observadas diferenças significativas entre mulheres negras e brancas, embora Leal et al. ${ }^{19}$ afirmem que, em relação à presença do companheiro na ida à maternidade, as gestantes pretas ficam em condição desfavorável em relação a brancas e pardas. Não foi possível aferir se a relação conjugal tem alguma influência na prática da violência obstétrica, dado que, nos estudos utilizados para esta revisão, não houve nenhuma análise desse dado.

Quanto à escolaridade, cumpre destacar que Leal et al. ${ }^{19}$ concluem que existem dois níveis de discriminação na sociedade - o educacional e o racial - e que ambos interferem na atenção oferecida pelo sistema de saúde. Acrescenta-se a essa afirmação o fato que acesso precário à educação se relaciona não apenas ao preconceito exteriorizado pelos profissionais como também à capacidade das mulheres em compreender as orientações recebidas e participar das decisões em relação a seu acompanhamento.

Todos os artigos analisados afirmam que não só as mulheres negras têm o pior atendimento à saúde como também possuem os piores índices de escolaridade e renda, embora se diferenciem na interpretação desse dado. Berquó e $\operatorname{Lago}^{\mathbf{2 0}}$, por exemplo, sugerem que o que interfere na saúde da mulher é sua condição econômica e não sua raça:

Os dados sugerem que mulheres com igual escolaridade, situação econômica e de posse (ou não) de plano de saúde têm chances similares de realizar o número mínimo de consultas de pré-natal, uma consulta de puerpério e ter acompanhante no trabalho de parto, independentemente de cor ${ }^{20(559)}$.

Por outro lado Domingues et al.12(290) ressaltam que este achado não permite afastar a existência de discriminação racial no acesso aos serviços de saúde reprodutiva, uma vez que o poder aquisitivo, mais do que outras características socioeconômicas, estaria captando esta mesma discriminação.

Essa discussão demonstra que falar sobre racismo não exclui a possibilidade de obter conclusões racistas.

Em relação à percepção do racismo sofrido, as mulheres negras, segundo Domingues et al. ${ }^{12}$, também reconhecem a diferença no tempo e qualidade dos atendimentos recebidos em comparação com as mulheres brancas. Para os autores, o reconhecimento do racismo pelas mulheres entrevistadas significa uma conquista na luta contra o racismo quando se leva em consideração o mito da democracia racial e sua força na sociedade brasileira.

A conclusão de que $o$ atendimento em saúde para as mulheres negras é precário foi quase unânime. Apesar de estarem longe do ideal, os índices de mortalidade materna e de acesso ao pré-natal melhoraram nos últimos 15 anos, consequência de um empenho iniciado pela implantação da Política Nacional de Saúde Integral da População Negra (PNISPN), além de outras políticas públicas que promoveram a redução das diferenças sócio demográficas e disparidades sociais ${ }^{21}$. Essa informação reforça o quão é necessário o contínuo debate a respeito do racismo junto à criação e efetivação de políticas públicas para essa população.

Por fim, apenas uma pesquisa analisa a relação entre os profissionais de saúde e a percepção do racismo institucional. Outras pesquisas analisadas mostraram a necessidade de se conscientizar e sensibilizar os profissionais de saúde em relação ao racismo, além de incluir em sua formação estudo mais aprofundado das doenças e enfermidades que acometem predominantemente a população negra.

É notável a presença do racismo institucional nas instituições de saúde, pois as mulheres negras continuam merecendo os piores índices de atendimento e sendo as maiores vítimas de 
violências obstétricas ${ }^{6,13}$. Confirmando, assim, o que foi trazido por Kalckmann et al. ${ }^{9}$, ao afirmarem que o racismo institucional atua em todos os âmbitos da saúde, ofertando à população as piores condições de atendimento, dificultando seu acesso a instituições e interferindo nas relações profissionais.

\section{Considerações finais}

O objetivo deste estudo foi levantar a produção científica no Brasil a respeito do racismo institucional e saúde da mulher negra e analisar como o tema tem sido tratado pelos pesquisadores. Chegou-se à conclusão que a escassa produção se configura como um reflexo do racismo institucional e pode ser vista nas barreiras impostas pelas instituições ao se tentar abordar o tema, o que dificulta a produção de pesquisas sobre o racismo. Logo, é necessário um maior envolvimento da academia com o tema de relações raciais e, consequentemente, maior produção de pesquisas.

Faz parte da estrutura racista brasileira o fortalecimento da crença na democracia racial. É comum observar a interpretação das desigualdades no atendimento e acesso à saúde da população negra como consequência da desigualdade econômica, mascarando, assim, o problema racial. Torna-se imprescindível que, além de maior produção científica sobre racismo, compreenda-se que associar a precariedade do atendimento à população negra com a sua condição econômica é reforçar o mito da democracia racial e, consequentemente, naturalizar o racismo.

A inclusão do quesito raça/cor nas bases de dados dos serviços trouxe consequências positivas para as produções científicas, mostrando ser uma ferramenta contra o racismo. Entretanto, cabe salientar que a inclusão dessa ferramenta sem uma conscientização da população a respeito do processo de identificação racial não é de total eficácia, tendo em vista que, em razão do processo de branqueamento e mestiçagem, a população brasileira tende a não reconhecer sua própria identidade racial.

A saúde da mulher negra continua sendo negligenciada em relação à da mulher branca, demonstrando a influência do racismo institucional nos processos de saúde e doença da população. A violência obstétrica é uma problemática que continua atual e sua predominância na população negra confirma a ação do duplo preconceito trazido por Domingues et al. ${ }^{12}$. O combate a essas desigualdades deve ser visto como prioridade; portanto, é necessário que o debate a respeito do racismo institucional seja constante nas instituições de saúde e entre os profissionais.

Conclui-se que o racismo institucional continua presente nas instituições de saúde, agindo de maneira implícita em todos os processos, seja nas relações profissionais ou no acesso ou atendimento à população negra. Logo, torna-se indispensável pensar a categoria raça e entender as especificidades da população negra para a obtenção de uma saúde igualitária.

\section{Colaboradores}

Oliveira BMC (0000-0002-2451-0916)* contribuiu para concepção, planejamento, análise e interpretação dos dados. Kubiak F (0000-0002-4077-919X)* contribuiu para a elaboração do rascunho e revisão crítica do conteúdo. 


\section{Referências}

1. Lima MEO, Vala J. As novas formas de expressão do preconceito e do racismo. Estud. Psicol. 2004; 9(3):401-411.

2. Nunes SS. Racismo no Brasil: tentativas de disfarce de uma violência explícita. Psic. USP. 2006; 17(1):8998.

3. Campo LA. Racismo em três dimensões. Uma abordagem realista-crítica. Rev. Bras. de Cien. Soc. 2017; 32(95):1-19.

4. Bernandino J. Ação afirmativa e a rediscussão do mito da democracia racial no Brasil. Est. Afro-Asiáticos. 2002; 24(2):247-273.

5. Guimarães FR, Newton PCC. Diz-se parda, para não se dizer negra: autodeclaração entre trabalhadoras domésticas sindicalizadas e não sindicalizadas do Brasil. Rev. Bras. de Direitos e Garantias Fundamentais. 2017; 3(2):128-143.

6. López LC. O conceito de racismo institucional: aplicações no campo da saúde. Interface- Comunic. Saúde e Educ. 2012; 16(40):121-134.

7. Albert V, Pereira AA. Movimento negro e "democracia racial” no Brasil: entrevistas com lideranças do movimento negro. In: III Conferência Bienal da Associação para o Estudo da Diáspora Africana Mundial; 2005 Out 5-7; Rio de Janeiro. Rio de Janeiro: CPDOC; p. 1-15.

8. Batista LE, Monteiro RB, Medeiros RA. Iniquidades raciais e saúde: o ciclo da política de saúde da população negra. Saúde debate. 2013; 37(99):681-690.

9. Kalckmann S, Santos CG, Batista LE, et al. Racismo Institucional: um desafio para equidade no SUS? Saúde Soc. São Paulo. 2007; 16(2):146-155.

10. Werneck J. Racismo institucional e saúde da população negra. Saúde e Soc. São Paulo. 2016; 25(3):535549.
11. Tavares NO, Oliveira LV, Lages SRC. A percepção dos psicólogos sobre o racismo institucional na saúde pública. Saúde debate. 2013; 37(99):580-587.

12. Domingues PML, Nascimento ER, Oliveira JF, et al. Discriminação racial no cuidado em saúde reprodutiva na percepção de mulheres. Texto Contexto Enferm. 2013; 22(2):285-292.

13. Lima KD. Raça e Violência Obstétrica no Brasil [monografia]. Recife: Centro de Pesquisas Aggeu Magalhães, Fundação Oswaldo Cruz; 2016. 25 p.

14. São Paulo. Defensoria Pública do Estado de São Paulo, Núcleo especializado de promoção a defesa dos direitos da mulher, Escola de Defensoria Pública do Estado de São Paulo. Violência Obstétrica: você sabe o que é? São Paulo: Defensoria Pública; 2014.

15. Gil AC. Como elaborar projetos de pesquisa. São Paulo: Atlas; 2010.

16. Cruz ICF. A sexualidade, a saúde reprodutiva e a violência contra a mulher negra: aspectos de interesse para a assistência de enfermagem. Rev. Esc. Enferm. USP. 2004; 38(4):448-457.

17. d'Orsi E, Bruggemann OM, Diniz SCG, et al. Desigualdades sociais e satisfação das mulheres com o atendimento ao parto no Brasil: um estudo nacional de base hospitalar. Cad. Saúde Pública. 2014; 30(supl1):154168.

18. Berlfort IKP, Kalckmann S, Batista LE. Assistência ao parto de mulheres negras em um hospital do interior do Maranhão, Brasil. Saúde e Soc. São Paulo. 2016; 25(3):631-640.

19. Leal MC, Gama GN, Cunha CB. Desigualdade raciais, sócio-demográficas e na assistência ao pré-natal e ao parto, 1999-2001. Rev. Saúde Pública. 2005; 39(1):100107.

20. Berquó E, Lago TDG. Atenção em saúde reprodutiva 
no Brasil: eventuais diferenciais étnico-raciais. Saúde e Soc. São Paulo. 2016; 25(3):550-560.

21. Diniz CSG, Batista LE, Kalckmann S, et al. Desigualdades sóciodemográficas e na assistência à maternidade entre puérperas no sudeste do Brasil segundo a cor da pele: dados do inquérito nacional Nascer do Brasil (2011-2012). Saúde e Soc. São Paulo. 2016; 23(3):561-572.

Recebido em 28/12/2018

Aprovado em 23/05/2019

Conflito de interesses: inexistente

Suporte financeiro: não houve 